\title{
Research on 3D Parametric Modeling Technology of Template Parts Based
}

\section{on CATIA}

\author{
Bei $\mathrm{CHEN}^{1}$, YanCAO ${ }^{2}$, Jiang $\mathrm{DU}^{3}$,MiaomiaoZHANG ${ }^{4}$ \\ Department of Mechanical and Electronic Engineering, Xi'an Technological University, \\ Xi'an, China \\ 1651723489@qq.com, ${ }^{2}$ jantonyz@163.com, ${ }^{3}$ Simen_du@sina.com
}

\begin{abstract}
With the object to improve the design efficiency and quality of general parts through the research and analysis of parametric modeling hierarchy, the parametric modeling technology is summarized. This method can be used to build reusable templates part based on CATIA, the research results of this paper can provide effective ideas and methods for the automation of part design with standardization and generalization.
\end{abstract}

Keywords:Parametric Modeling; Design Reuse; CAD

\section{Introduction}

CATIA is a CAD/CAE/CAM integrated software, which is widely used in aerospace, automobile manufacturing, shipbuilding, machinery manufacturing, electronics/electrical appliances, consumer goods and other industries. 3D modeling and modeling can be directly used in CATIA V5 software.

Parametric modeling is the problem which has been explored by CAD designers, the key is how to use the physical characteristics of parameters to automatically control and generate real 3D model, and feature parameters can be automatically changed to reflect the three-dimensional model. However, different parts of the parametric design method are not exactly the same, some parts can be directly completed based on the known structural parameters. But most of the parts are containing one or more unknown parameters, At the same time, it is necessary to achieve the parametric design model based on the traditional method modeling and further according to the design requirements. Based on the above analysis, the parametric modeling of parts can be divided into three levels as shown in Figure 1 below $^{[1]}$.

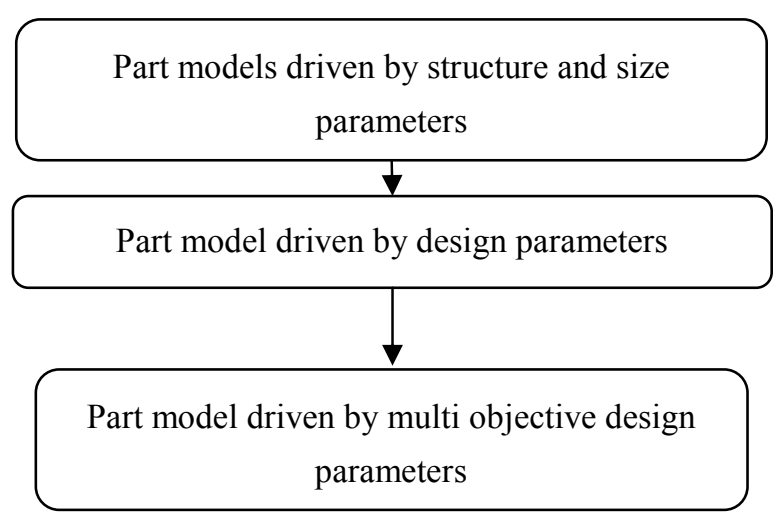

Figure1Classification of parametric modeling parts

According to the different standard parts and common parts, the parameterized template of the part is established by using the parametric modeling technology of corresponding level in the above figure. 


\section{CATIA parametric modeling method}

The key to parametric modeling is to use parameters, formulas, tables, features and other driving graphics to achieve the purpose of changing graphics, in CATIA V5, it can be achieved through the following methods ${ }^{[2]}$.

(1)The model is driven by system parameters and size constraints

CATIA V5 has a perfect function of automatic extraction system parameters. When the sketch is designed, the size constraint input by designer is saved as the characteristic parameter, and it is modified visually in the subsequent design so as to achieve the purposes to create parameters modeling directly. The key to driving the graphics with the system parameters is how to convert the parameters extracted from the object into CATIA to control the characteristic parameters of the 3D model. Size-driven is the basis of parameter-driven, dimension restriction is the premise to achieve size-driven.The characteristics of CATIAV5 dimension constraint is to combine the shape and size, and to realize the control of the geometry by the dimension constraint. The complete size parameter must be considered in the design as the starting point (full constraint), the size(under-constraint) can't be leak, the size cannot be marked(over constraint). After the graph is completely constrained, its size and position relationship can change in coordination, and the system will directly convert the size constraints into system parameters. So as to realize the ideal parameterized driven model.

(2)User parameters and formula-driven models

Designers can use the user-defined parameters and formatting tools, can easily customize the various parameters and the relationship between the parameters. Parameters such as geometric parameters (such as length, angle, etc.), physical parameters (such as material, mass, density, temperature, etc.), dimensionless parameters (such as integers), Boolean parameters and string parameters and so on. By using the formula, we can realize the linkage between various parameters, and add a variety of relationships between parameters. After the user parameter definition, the user parameters and system parameters must use the formula to establish appropriate relationships to achieve the model of the driver, through the drive system parameters to drive the model indirectly, therefore the user parameters and formulas are inseparable.

(3)The model is driven by tabular data

For a series of products (such as most standard parts and common parts), these models often have the same topology, but some of the sizes are different, these key sizes can be obtained through the table search. CATIA V5 provides the function to associate parameters with tabular data. The data related to the model in the form of tables are stored in the corresponding file, and then use the "design table" tool to model the parameters (which can be system parameters can also be user parameters) and associated with the data in the table, the model can be driven by selecting different data to achieve in the design table tool. There are two charts can be selected, one is the text format of the chart file, another is the excel format of the chart file. The two charts through the CATIA V5 tool "design table" to access the records of each table, so as to modify the size and change the shape of the objective.

\section{The establishment of parametric part template}

The model is established by using the size constraints and geometric topology constraints in the sketch editor to establish sizes and their relationships. When the part structure parameter size table and the 2D part structure diagram are known, this paper uses the method of table data to drive the graph and to create the parametric model. According to figure 1, three kinds of parametric models was established with three typical part such as"gasket and hexagon bolts", which are modeled by selecting the method of "user parameter and formula-driven graph" and the mechanism of ideological 3D parametric modeling system is analyzed under different conditions. 


\subsection{Parametric modeling of part template driven by structure and dimension parameters}

According to the structure data of gasketdefined in GBT 1230-2006, the 3D parametric modeling is carried out. CATIA model document type is divided into part and product, representing parts and components, the parts are selected here to create. Open CATIA V5 and enter the part design block. There are the basic parameters of the gasket: outside diameter (d2), gasket thickness (h), gasket diameter (d1), gasket inner diameter (d3) and so on, the above parameters are created in the formula as shown in Figure 2 below.

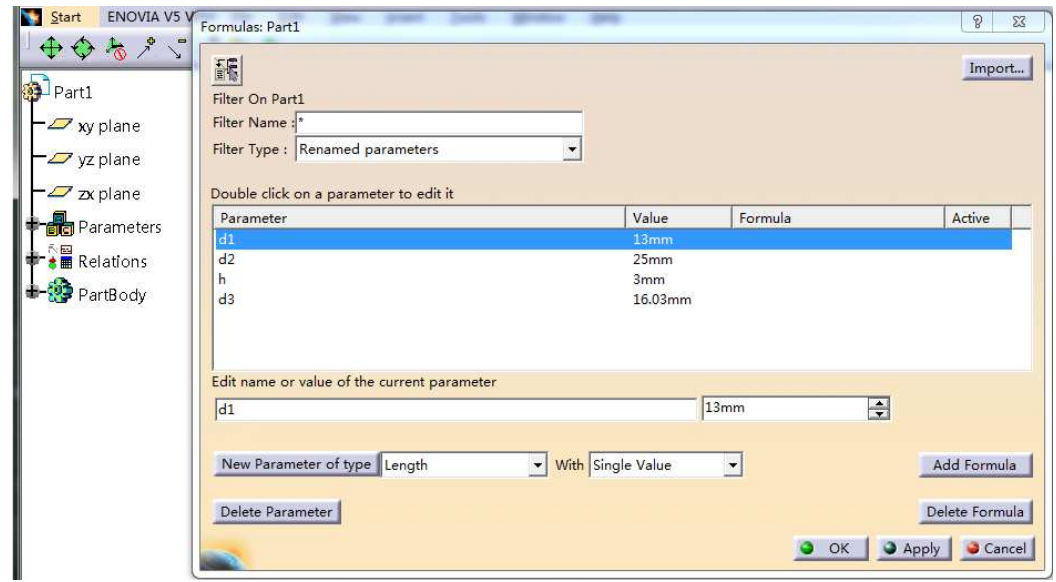

Figure2 CATIA gasket parameters in the set display.

Draw the sketch of the gasket in the part design module, then we constrain and parameterize the sketch by the formula, and then get the model of the gasket by drawing command, groove command and chamfer command, as shown in Figure. 3 and Figure.4. By modeling the gasket, recognized how parts of the component are modeled when all the structural parameters of the part are known.

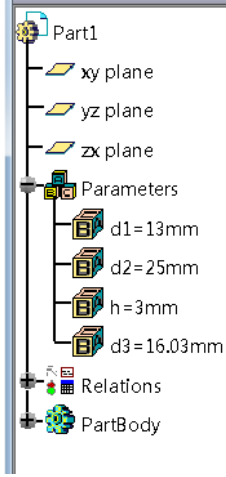

Figure 3 User parameters in the model tree

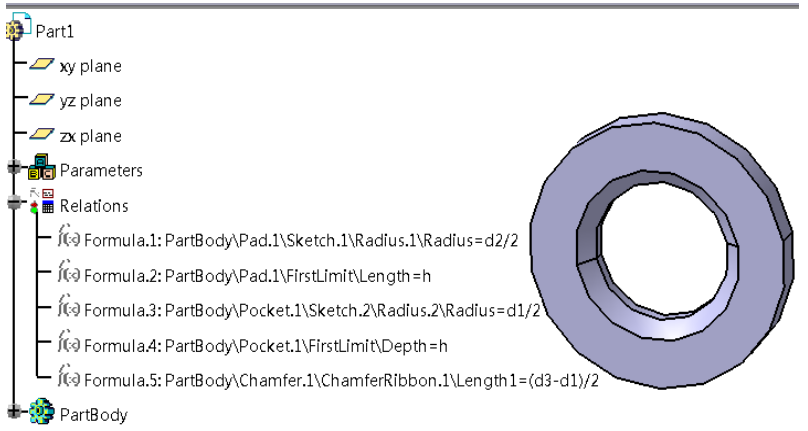

Figure 4 Parameter relation in model tree

\subsection{Parametric modeling of part template driven by design parameters}

In the parametric modeling of the part, if one design parameter is unknown, it can be calculated by another structural parameter with formula relationship. In fact, parts of the design objectives and the use of requirements are mainly embodied in the "load, deformation" and other aspects. The bolts of heavy machine parts in GB/T27-2013Fa2, only consider the bolt's squeezing force, the function parameters of the hexagonal head bolts are subject to working shear force $F$, extrusion strength $\sigma_{p}$. The relationship between the above three functional parameters and the other structural parameters of the bolt Shows in table 1. 
Table 1 Bolt parameters of squeeze pressure

\begin{tabular}{|c|c|c|c|}
\hline Bolt type & Function parameter & Structure parameter & $\begin{array}{l}\text { Relation and calculation } \\
\text { formula }\end{array}$ \\
\hline $\begin{array}{l}\text { Bolt with } \\
\text { working shear }\end{array}$ & $\begin{array}{l}\text { Maximum compressive } \\
\text { stress of bolt } \sigma_{\mathrm{p} \max }\end{array}$ & $\begin{array}{l}\text { Bolt diameter } \mathrm{d} \\
\text { Six angle bolt head to edge } \\
\text { width } \mathrm{S} \\
\text { Six angle bolt head } \\
\text { thickness } \\
\text { Pitch } \mathrm{P} \\
\text { Fillet r,Length of bolt } 1 \\
\text { Bolt rod and hole wall } \\
\text { extrusion surface } \\
\text { Height } \mathrm{l}_{3} \\
\text { The length of the bolted } \\
\text { bar } \mathrm{l}_{2} \\
\text { Bolt reinforcement bar } \\
\text { diameter dp }\end{array}$ & $\begin{array}{l}\sigma_{p}=\frac{F}{d_{0} L_{\min }} \leq\left[\sigma_{\mathrm{p}}\right] \\
L_{\min } \geq 1.25 d_{0}\end{array}$ \\
\hline
\end{tabular}

Note: $\left[\sigma_{\mathrm{p}}\right]$ : bolt material allowable compressive stress, MPa

After the known bolt material, the allowable extrusion stress $\left[\sigma_{\mathrm{p}}\right]$ can be checked in the mechanical manual and entered into the formulas in the table, take the extrusion stress $\sigma_{\mathrm{p}}$ is equal to the maximum [ $\left.\sigma_{\mathrm{p}}\right]$, according to Table 1 in the two formulas, the bolt diameter of the shear surface $\mathrm{d} 0$ which equal to the bolt diameter $\mathrm{d}$ can be calculated when the extrusion is maximum, it also can calculatethe $\mathrm{L}_{\min }$ which equal to the bolt and the hole wall extrusion surface height 13.After calculated the $\mathrm{d}$ and 13 , other structural parameters can be obtained by the parameters of the query bolt shown in Figure 5.

\begin{tabular}{|c|c|c|c|c|c|c|c|c|c|c|c|}
\hline PartNumber & $d(m m)$ & $P(\mathrm{~mm})$ & $\mathrm{ds}(\mathrm{mm})$ & $\mathrm{S}(\mathrm{mm})$ & $\mathrm{K}(\mathrm{mm})$ & $r(m m)$ & $\mathrm{dp}(\mathrm{mm})$ & $12(\mathrm{~mm})$ & $e(m m)$ & $\mathrm{l}(\mathrm{mm})$ & $13(\mathrm{~mm})$ \\
\hline gbt27-2013FA2_1 & 14 & 2 & 15 & 21 & 8 & 0.6 & 10 & 3 & 23.35 & 40 & 15 \\
\hline gbt27-2013FA2_2 & 14 & 2 & 15 & 21 & 8 & 0.6 & 10 & 3 & 23.35 & 45 & 20 \\
\hline gbt27-2013FA2_3 & 14 & 2 & 15 & 21 & 8 & 0.6 & 10 & 3 & 23.35 & 50 & 25 \\
\hline gbt27-2013FA2 4 & 14 & 2 & 15 & 21 & 8 & 0.6 & 10 & 3 & 23.35 & 55 & 30 \\
\hline gbt27-2013FA2_5 & 14 & 2 & 15 & 21 & 8 & 0.6 & 10 & 3 & 23.35 & 60 & 35 \\
\hline gbt27-2013FA2 6 & 14 & 2 & 15 & 21 & 8 & 0.6 & 10 & 3 & 23.35 & 65 & 40 \\
\hline gbt27-2013FA2_7 & 14 & 2 & 15 & 21 & 8 & 0.6 & 10 & 3 & 23.35 & 70 & 45 \\
\hline gbt27-2013FA2_8 & 14 & 2 & 15 & 21 & 8 & 0.6 & 10 & 3 & 23.35 & 75 & 50 \\
\hline gbt27-2013FA2 9 & 14 & 2 & 15 & 21 & 8 & 0.6 & 10 & 3 & 23.35 & 80 & 55 \\
\hline gbt27-2013FA2_10 & 14 & 2 & 15 & 21 & 8 & 0.6 & 10 & 3 & 23.35 & 85 & 60 \\
\hline gbt27-2013FA2_11 & 14 & 2 & 15 & 21 & 8 & 0.6 & 10 & 3 & 23.35 & 90 & 65 \\
\hline gbt27-2013FA2_12 & 14 & 2 & 15 & 21 & 8 & 0.6 & 10 & 3 & 23.35 & 95 & 70 \\
\hline gbt27-2013FA2_13 & 14 & 2 & 15 & 21 & 8 & 0.6 & 10 & 3 & 23.35 & 100 & 75 \\
\hline gbt27-2013FA2_14 & 14 & 2 & 15 & 21 & 8 & 0.6 & 10 & 3 & 23.35 & 110 & 85 \\
\hline gbt27-2013FA2_15 & 14 & 2 & 15 & 21 & 8 & 0.6 & 10 & 3 & 23.35 & 120 & 95 \\
\hline gbt27-2013FA2_16 & 14 & 2 & 15 & 21 & 8 & 0.6 & 10 & 3 & 23.35 & 130 & 105 \\
\hline gbt27-2013FA2_17 & 14 & 2 & 15 & 21 & 8 & 0.6 & 10 & 3 & 23.35 & 140 & 115 \\
\hline
\end{tabular}

Figure 5 Bolt parameter list

In CATIA, the structural parameters of boltscan be established in Figure 6, the bolt will be constrained and parametric the draft through the formula in the draft of bolt with the part design module, and then the bolt can be built parametric modelingusing the command of stretch, groove, thread, chamfering and so on, it is shown in Figure 7. 


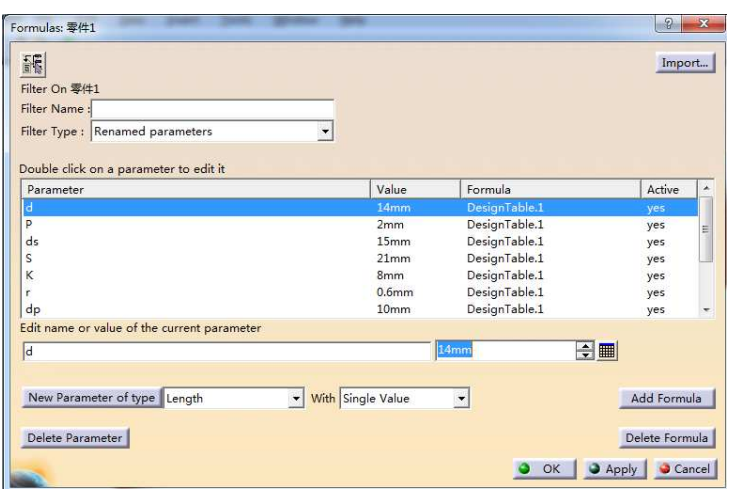

Figure6 Setting of bolt parameters

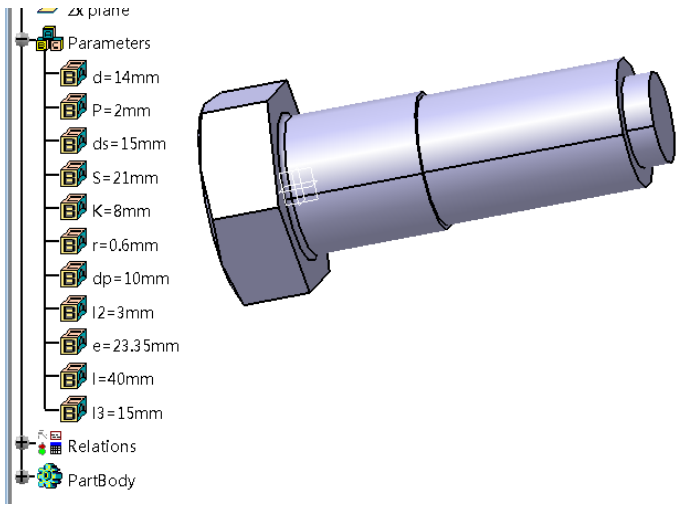

Figure7 Parametric modeling of bolts

\subsection{Parametric modeling of hexagon bolts}

Hexagon bolt is a frequently used components, when we begin modeling,we should first consider the bolt in the actual design goals and requirements of the main expression of "extrusion, shear" and other aspects.The corresponding hexagonal bolts can be defined as the function parameters: The working shear force on the bolt $\mathrm{F}$, extrusion strength $\sigma_{\mathrm{p}}$ and shear strength $\tau$. Table 1 shows the relationship between the above three functional parameters and the other structural parameters of the bolt.

Table 2 Bolt parameters for extrusion and working shear

\begin{tabular}{|c|c|c|c|}
\hline Bolt type & Function parameter & Structure parameter & $\begin{array}{l}\text { Relation and calculation } \\
\text { formula }\end{array}$ \\
\hline $\begin{array}{c}\text { Bolt with } \\
\text { working shear }\end{array}$ & $\begin{array}{l}\text { Working shear force of bolt } \\
F \\
\text { Maximum compressive } \\
\text { stress of bolt } \sigma_{\text {pmax }} \\
\text { Maximum shear strength of } \\
\text { bolt } \tau_{\max }\end{array}$ & $\begin{array}{l}\text { Bolt diameter } d \\
\text { Six angle bolt head to edge } \\
\text { width } \mathrm{S} \\
\text { Six angle bolt head } \\
\text { thickness } \\
\text { Pitch } \mathrm{P} \\
\text { Fillet } \mathrm{r} \text {, Length of bolt } 1 \\
\text { Bolt rod and hole wall } \\
\text { extrusion surface } \\
\text { Height } \mathrm{l}_{3} \\
\text { The length of the bolted } \\
\text { bar } 1_{2} \\
\text { Bolt reinforcement bar } \\
\text { diameter dp }\end{array}$ & $\begin{array}{l}\sigma_{p}=\frac{F}{d_{0} L_{\min }} \leq\left[\sigma_{\mathrm{p}}\right](1) \\
\tau=\frac{\mathrm{F}}{\frac{\pi}{4} \mathrm{~d}^{2}} \leq[\tau](2) \\
L_{\min } \geq 1.25 d_{0}(3)\end{array}$ \\
\hline
\end{tabular}

Note:[ $\tau]$ :Allowable shear stress of bolt material, $\mathrm{MPa} ;\left[\sigma_{\mathrm{p}}\right]$ :bolt material allowable compressive stress, $\mathrm{MPa}$

According to the relationship between the hexagonal bolt function and the structural parameters shown in Table 1, after the bolt material is defined, the allowable extrusion stress $\left[\sigma_{p}\right]$ and the allowable shear stress $[\tau]$ of the bolt material are searched from the mechanical manual. Bringing the two parameters to the table, the maximum value of the compressive stress $\sigma_{\mathrm{p}}$ is equal to $\left[\sigma_{\mathrm{p}}\right]$, and the maximum shear stress $\tau$ is equal to [ $\left.\tau\right]$. The parametric drive-based design of the bolt part can be modeled in the following four steps:

1) In actual engineering, if the working load $\mathrm{F}$ of the bolt is known, then the diameter $\mathrm{d} 01$ of the bolt under the pressing force is calculated by using the formulas 1 and 3 in Table 2. 
2) The bolt under the shear force can be calculated the diameter d02 and the size Lmin which is equal to the bolt and the hole wall extrusion height 13 with the second formula in the table 2.

3)Through the above steps, the diameter of the two shear surface of the bolt can be calculated. so the diameter of the bolt $\mathrm{d}=\max \{\mathrm{d} 01, \mathrm{~d} 02\}$, other structural parameters can be get by querying the bolt parameters shown in Figure 5.

Open CATIA, building the structure parameters in the formula command of toolbar has been described above in detail, without concrete description in Figure 6. In the part design module, drawing the sketch of the bolt, constraining and parameterizing the bolt by the formula. The hexagonal bolt model is created by the operation such as "drawing, rotating, and thread", as shown in Figure 8 below.

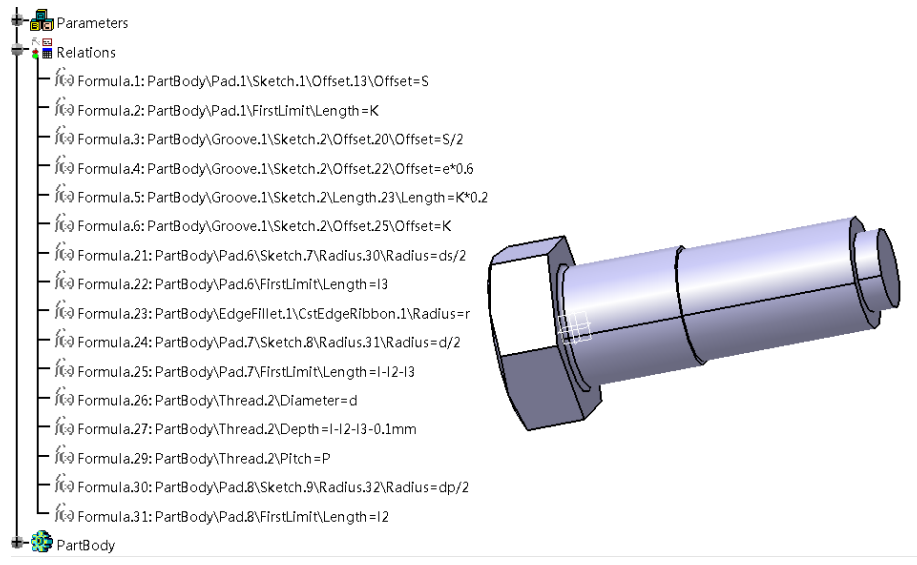

Figure8 Modeling of hexagon bolts

Through the above three examples, showing that the three parts of the modeling steps can be carried out in accordance with the process figure 9.

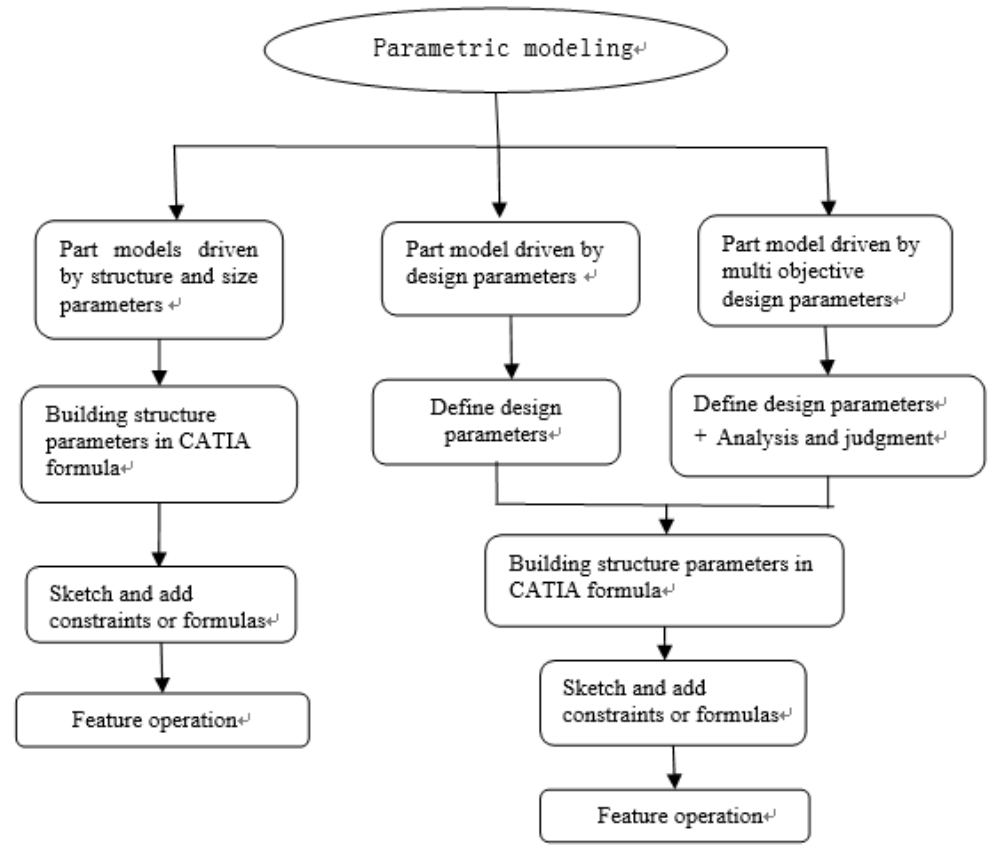

Figure9 Part parametric modeling process 


\section{Summary and Prospect}

This paper describes the method of establishing 3D parametric model, using the modeling of "user parameters and formulas of driving pattern" based on CATIA V5 software. By creating a sketch based feature and transformation feature to establish three levels of different types of parts of the model, and the basic parts of the modeling process is summarized. Parametric modeling is a core work in the establishment of standard parts library. It can greatly reduce people's workload and improve work efficiency for serial products design such as standard parts, it has very great practical value. In addition, if the CATIA parametric modeling technology and its secondary development technology are combined, it would be achieved more and more complex functions, there will be a great application value. Future parts model to study may be the intelligent part model with multi-design objectsand multi-constraints, it needsus to study and exploration more.

\section{Acknowledgments}

The paper is supported by Key Problem Tackling Project of Shaanxi Scientific and Technological Office (2016GY-024).

\section{References}

[1] Guo Jing, Zhao Guangdong. Parametric modeling and design of 3D parts[J]. Premiere, 2015,12:28.

[2] Wang Xiaoyou.3D Parametric Modeling Method and Application Based on CATIA[J].Auto Parts, 2012,03:55-58.

[3]Shen Yanhui, Zou Linghua, Dai Liqiang, Zhao Xingyang, Zhao Fuquan. Parameterized Design and Database Set-up of Standardized Parts Based on CATIA[J].Auto Engineer, 2012,09:53-55.

[4]Wang Yonggang, Yang Lijie. Three-dimensional Parametric Modeling Technology and Application Based on CATIA[J]. Mechanical Engineer, 2014,05:200-202.

[5]Li Jinwang. Parameterized Design Study on Mechanical Devices[A]. Electronic mechanical engineering branch of China Electronic Society.Proceedings of the Conference on Mechanics and Electronics[C]2011.2011:5.

[6] Pu Lianggui, Chen Guoding, Wu Liyan.Mechanical Design Ninth Edition[M]. Xi'an: Higher Education Press, $2012,08$.

[7]Liang Zhengang, Sun Guodong. 3D parametric modeling technology for aircraft parts based on CATIA/CAA[J]. Journal of Sichuan Ordnance,2012,03:72-74.

[8] Sun Guodong.3D Parametric modeling and Data management technology Based on definition[D].ShenyangLigongUniversity,2012

[9]JorgeD.Camba,ManuelContero,PedroCompany.ParametricCADmodeling:Ananalysisofstrategiesfordesignreusability[J].C omputer-AidedDesign, 2016.

[10]YongmingWang,JiangtaoLi.UMLModelingandParametricDesignforCrossShaftUniversalCouplingCADSystem[J].Journa lofSoftware,2012,79. 